Fecha de recepción: diciembre 2019

Fecha de aceptación: febrero 2020

Versión final: marzo 2020

\title{
Cuerpos que importan. Reflexionando sobre el estado actual de la industria del denim y las problemáticas que contiene
}

Alexandra Louise Vinlove ${ }^{(1)}$

Resumen: En la actualidad se ha vuelto evidente la necesidad de sostener un cambio de paradigma en los sistemas de producción y comercialización de la industria del denim, trabajando a partir de conceptos como la economía circular y el Diseño para la Transición (Irwin, Kosoff y Tonkinwise, 2015) con la finalidad de convertir a la moda en una práctica sostenible. A partir de un análisis crítico del escenario y utilizando el marco conceptual del Diseño para la Transición se busca aportar ideas para una propuesta de intervención utilizando los puntos de apalancamiento propuestos por Donella Meadows (1997).

Palabras clave: Diseño para la Transición - Leverage Points - Wicked Problems - Economía Circular - Industria del Denim - Diseño de Indumentaria - Diseño Sostenible - Sostenibilidad.

[Resúmenes en inglés y portugués en las páginas 162-163]

(1) Alexandra Louise Vinlove es Diseñadora de Indumentaria, graduada en 2016 de Técnica en Diseño de Moda e Indumentaria de la Asociación Biblioteca de Mujeres (ABM) y Licenciada en Diseño de la Universidad de Palermo (2019). Desde el año 2017 se desempeña como Asistente de Docencia para la Cátedra de Delia Cancela, dentro de la carrera de Diseño de Moda e Indumentaria en ABM. Ejerce como diseñadora independiente con especialización en diseño sostenible, habiendo presentado alguno de sus trabajos en la muestra estática titulada Camino a la sustentabilidad, exhibida para la Semana de la Moda 2019, BA Moda, Buenos Aires Ciudad (CMD). En julio de 2019 se presentó como expositora en el marco del XIV Encuentro Latinoamericano de Diseño, llevado a cabo por la Universidad de Palermo, dictando una conferencia sobre diseño sostenible titulada Revestir: Fashion Upcycling. allyvinlovedesign@gmail.com

\section{Introducción}

En la actualidad se ha vuelto evidente la necesidad de sostener un cambio de paradigma en los sistemas de producción y comercialización de la industria del denim, trabajando a partir de ideas y conceptos relacionados con la economía circular y el Diseño para la Transición 
(Irwin, Kosoff y Tonkinwise, 2015) con la finalidad de convertir a la moda en una práctica sostenible. A partir de un análisis crítico del escenario y utilizando el marco conceptual del Diseño para la Transición se busca aportar ideas para una propuesta de intervención utilizando los puntos de apalancamiento propuestos por Donella Meadows (1997).

El caso analizado aborda las diferentes problemáticas que se desenvuelven dentro de esta industria, principalmente la falta de diversidad en los talles y la resultante objetualización y alienación de los cuerpos femeninos, además de los impactos derivados de sus prácticas productivas sobre el medioambiente y consecuente pérdida de biodiversidad.

Esta propuesta parte de la utilización del Diseño para la Transición como lente para el análisis, y como herramienta para recontextualizar el presente y el futuro de este sistema, donde el diseñador puede actuar como agente de cambio, con la finalidad de generar modificaciones en los procesos de producción y en las conductas de sus consumidores. De esta manera, se hace foco en la relación entre -sociedad, economía y ambiente- donde la identidad de los individuos se ha vinculado estrechamente con el consumo, por lo que se busca diseñar una intervención que englobe perspectivas de sostenibilidad tanto en lo ético como en lo ambiental.

A través de esta propuesta se plantea orientar la Gestión de Diseño hacia la promoción de algunos de los Objetivos de Desarrollo Sostenible (ONU), relacionados con (a) la sostenibilidad social: con la igualdad de género, la reducción de las desigualdades, el trabajo en condiciones decentes, el consumo responsable; y en lo vinculado con (b) la sostenibilidad ambiental: se busca la revalorización del diseñador como productor responsable, para generar el saneamiento del agua, la utilización de energías renovables y no contaminantes, la acción por el clima y los ecosistemas terrestres, la vida submarina y ciudades sostenibles. Utilizando estos objetivos como una guía para la gestación de visiones de futuro, se busca contribuir a la creación de nuevos modelos socio-técnicos como forma de transición de largos horizontes de tiempo, hacia un futuro sostenible.

A continuación se presenta una síntesis de algunos de los apartados analizados:

\section{El Denim y las tendencias}

Volviendo la mirada sobre el caso que se analiza, el estado actual de la industria del denim muestra evidencias relativas con la insostenibilidad de sus prácticas, ya que conducen a la exclusión, al aumento de las desigualdades, condiciones de trabajo insalubres, la agresión medioambiental y el agotamiento de la biodiversidad. Luego de estudiar el ciclo de obsolescencia programada en una pieza de denim y analizar los principales impactos y sus causas relacionadas con la industria y el consumo, se elabora un mapeo de Wicked Problem del sistema que lo contiene (Rittel y Webber, 1973), que permite identificar a los actores intervinientes y detectar los posibles puntos de intervención dentro del sistema. Trabajando principalmente con tres de los puntos de apalancamiento (Leverage Points) propuestos por Donella Meadows (1997) se elabora y presenta el rediseño del ciclo de vida del producto, creando una nueva experiencia de comercialización inclusiva, que incorpora principios de economía circular como propuesta de transición hacia las visiones de futuro sostenible. 
A medida que se modifican y evolucionan las necesidades, los hábitos y las preferencias de los consumidores en función de los avances en la tecnología y la comunicación, también lo hacen las industrias para poder responder con mayor rapidez a estos cambios y así continuar con la generación de demanda y el establecimiento del consumo como estilo de vida. Este fenómeno se desenvuelve dentro del marco de la modernidad líquida (Bauman, 2000) y la conformación de la sociedad de consumo (Vizcarra y Ovalle, 2011), que se caracteriza por su naturaleza ambivalente y en constante cambio, en la que se pueden identificar tres variables de curso constante: (a) la búsqueda de identidad en un territorio incierto, (b) la interconectividad humana a partir de la aparición de la tecnología digital, y (c) la presencia de fuertes límites medioambientales y la pérdida de biodiversidad.

Estas variables se ubican dentro de una sociedad considerada hiperconsumista, donde la moda, entendida como una tendencia, promueve un anclaje entre la identidad de los individuos y el consumo a través de una constante generación de demandas y la implementación de ciclos de obsolescencia programada. Tal como elabora Cline,

(...) como la moda se expresa públicamente, todos pueden ver quién no se encuentra alineado; y por eso, para mantenerse al día con los estilos más recientes, tienen que comprar de manera constante (Cline: 2014, 11).

Estos conceptos conforman la realidad sociocultural en la que el diseño se desempeña como actividad, como agente y como herramienta en la actualidad. Los procesos de producción y comercialización y las prácticas de consumo que se desprenden del diseño de moda e indumentaria, particularmente dentro la industria del denim, se analizan en función de su relación con la identidad de los individuos y de su impacto sobre la sociedad y el medioambiente, con la finalidad de proponer alternativas con perspectiva sostenible. Se trabaja tomando como objeto de estudio principal a las prácticas del diseño de indumentaria, partiendo del estrecho vínculo que tiene la indumentaria con la identidad de los individuos pertenecientes a una determinada sociedad y sus estilos de vida. La vestimenta cumple la función de abrigo y protección del cuerpo, pero también de objeto decorativo y de herramienta de comunicación no verbal en la representación de las creencias y los valores del portador, además de transmitir su poder adquisitivo, sus intereses y su estilo de vida.

Es tal el grado de identificación entre cuerpo y vestido que una modificación en la ropa o indumento implica siempre una variación actitudinal. El vestido es una expresión indirecta de cada individuo, y no sólo está incluido en forma definitiva en nuestra propia imagen, sino que es parte de la imagen del otro (Pampliega de Quiroga y Pichon-Riviere, 1985:40). Debido a este importante rol que cumple la vestimenta en la vida de los individuos y dentro de la sociedad contemporánea, es que se produce (como uno de varios sus factores causales) el fenómeno de la moda rápida o fast fashion, dentro de una industria con alcance globalizado, en constante cambio y donde participan diversidad de actores: desde diseñadores a los consumidores junto a incontables representantes y colaboradores que cumplen una miríada de roles. La industria de la moda vuelve descartable y fácilmente reemplazable a los productos de la indumentaria, en una búsqueda constante de novedad que depende de la interconectividad del contexto, dentro de un mundo globalizado, don- 
de se integran determinados productos culturales a la industria del consumo, entendiendo que el producto cultural dentro del imaginario colectivo se conforma por costumbres, íconos y valores sociales connotados.

A partir de un estudio que trata acerca del surgimiento de nuevas tendencias de consumo identificadas para el año 2025 llevado a cabo por el Observatorio de Tendencias del INTI, se observan en sus resultados, cambios en las conductas de los individuos y sus estilos de vida, movilizados por una aumentada consciencia social y ambiental. Estos cambios se reflejan en la búsqueda de mayor igualdad de género, mayor libertad en la expresión de la identidad, en una tendencia hacia las representaciones auténticas de diversidad y experiencias de consumo personalizadas, manteniendo como lente principal a la sostenibilidad. Actualmente las marcas con mayor éxito ya no apuntan a un público universal, sino que buscan ir más allá de las clásicas segmentaciones por sexo, edad y/o clase social, poniendo su foco en una clasificación según estilos de vida. Sin embargo, las modas contemporáneas asociadas al diseño de indumentaria no siempre muestran interés en acompañar estos cambios ni mucho menos fomentarlos, al mantener intactas ciertas estructuras de producción y consumo desmedido, continuando además con la propagación de estereotipos limitantes comercializados a través de los medios de comunicación masiva.

\section{El Denim y la ley de talles en Argentina}

(...) La moda actúa igual que una disciplina que dociliza los cuerpos; y estos una vez cautivados por sus telas de araña, se visten según sus mandatos. El mayor riesgo de la moda es que atenta contra la cultura, ya que la hace homogénea y facilita las condiciones para una sociedad más totalitaria e inhumana (Croci y Vitale, 2012: 34-35).

El orden visual (entre otros actores) intervino de manera histórica, y en la manera en que el sujeto de la modernidad ha conocido y representado su propio cuerpo. Ha determinado los modos de tipificar los cuerpos de las personas y de interpretar lo social. A través de la dimensión corporal se ejerce la regulación y el control social de los sujetos, la sociología ha interpretado las prácticas del vestir y en especial en el universo de la moda, como hechos sociales que ponen en evidencia la construcción cultural de la conformación social de los cuerpos (Zambrini, citado en Figari, Jones y Pecheny, 2008: 142). De esta manera se evidencia que el comportamiento $y$, por ende, la vestimenta de los individuos queda sujeta al poder de las instituciones y medios de comunicación, haciendo aparente el poderoso e importante rol de la moda en la vida de los individuos, donde urge la necesidad de convertir esta industria en otra regulada por principios de inclusión, diversidad, y de cuidado y respeto por el medioambiente.

Para dar cuenta de las problemáticas que se desprenden de estas estructuras que muy a menudo se impregnan dentro del funcionamiento de la moda y, en este caso puntual, dentro la industria del denim, se plantean algunos datos relevantes recopilados de una encuesta realizada por la Asociación AnyBody Argentina en el año 2016. 
- Actualmente en la Argentina circulan 14 leyes de talles provinciales y regionales en vigencia, difiriendo entre sí en distintas y múltiples instancias, pero aun así todavía no existe una ley nacional de talles que cumpla el rol de regular consistente y uniformemente el actuar de las empresas productoras dentro del rubro de textil e indumentaria.

- Debido a esto es que, en general, las marcas operan de forma independiente, instaurando su propio rango de talles de manera casi aleatoria, basándose principalmente en el establecimiento de medidas que les permitan recortar gastos de producción y comercialización para aumentar su margen de ganancias.

- Debido a estas notables diferencias en las tablas de talles dentro del mercado y la consecuente falta de diversidad existente, se ha detectado que 7 de cada 10 argentinos no consiguen su talle real al momento de realizar las compras, considerando que el 95\% de esos consumidores son mujeres.

- De la misma manera, el $67 \%$ considera que su talle es dos veces mayor a lo que perciben como el ideal reflejado en las campañas publicitarias de las marcas. En consecuencia, el $80 \%$ de estos consumidores creen que deben adelgazar para poder conseguir ropa y para sentir que se asemejan más a lo que consideran socialmente aceptado o promovido.

A partir de los datos mencionados, se detalla información numérica relevante sobre la faltante de talles en la industria del denim en Argentina.

- En promedio, las marcas dominantes dentro del mercado tienen 7 talles regulares y 5 talles grandes o especiales, con una diferencia de 33 centímetros entre el menor y mayor talle regular y un margen de diferencia de sólo 26 centímetros entre el menor y el mayor talle especial.

- Además, el sistema de comercialización de estos talles es frecuentemente diferenciada, significando que estas marcas, exhiben casi exclusivamente los talles regulares, obligando a quiénes no se ajustan a esas medidas a comprar en locales de segunda mano, fuera de los circuitos de comercio comunes.

Finalmente la clasificación estética del mundo supone una permanente tensión y negociación respecto de cuáles identidades son susceptibles de ser visibilizadas legítimamente y cuáles se transforman en diferencias u otredades. Porque las prácticas del vestir, en tanto discurso estético, hacen referencia a la comunicación y clasificación social de los cuerpos generizados (Zambrini, citado en Figari, Jones y Pecheny, 2008: 144).

\section{El Denim y los procesos productivos en Argentina}

En lo referente a los procesos productivos de la industria del denim en Argentina, se advierte que la mayoría de las plantas de producción textil se localizan en las provincias de Chaco, Corrientes y Santa Fe, donde usualmente los costos de infraestructura y mano de obra son menores. A cada prenda virgen se le aplican entre 7 y 15 procesos, desde lavados a teñidos y desgastes, llevando a un tiempo de elaboración de aproximadamente 70 días para cada prenda en su totalidad. (Finalmente, el precio de venta promedio a la fecha de cada 
prenda de la tipología de pantalón jean, por ejemplo, es de un rango de 700 pesos argentinos como mínimo y de aproximadamente 5000 pesos argentinos como máximo)

Se considera que los principales impactos sociales y ambientales de estos sucesos, son

- discriminación por falta de inclusividad en las tablas de talles

- segregación en la experiencia de compra debido a la comercialización diferenciada

- prendas con un ciclo de vida corto por producir ahorros en la línea de producción

- generación de residuos textiles sólidos por descarte y

- una comercialización general basada en los principios de una economía lineal.

Teniendo en cuenta lo que desarrolla Cline, la moda rápida influye enormemente en el comportamiento de los individuos, pero

(...) muestra muy poco interés por el medioambiente y los derechos humanos. Reconstruyó toda la industria textil y del vestido a su imagen y semejanza: cambió de manera muy profunda la idea que tenemos del vestir... (Cline: 2014, 12).

Un claro ejemplo del impacto de la moda sobre la biodiversidad y de su participación en el agotamiento de los recursos naturales, parte de un anuncio de la Comisión Económica de las Naciones Unidas donde se considera

- que la industria de la moda es el segundo usuario de agua más grande del mundo y en lo referente a la generación de residuos se considera

- que el 85 por ciento de los textiles quedan en desuso y son enviados a los rellenos sanitarios de distintos países periféricos a nivel global.

Los datos anteriormente expuestos reflejan cómo la moda a menudo es restrictiva y acarrea una serie de efectos negativos por sobre un grupo de la sociedad, en lo particular y muy frecuentemente, las mujeres y comunidades minoritarias.

Partiendo del Diseño para la transición como posible catalizador hacia un cambio de paradigma en la gestión de los procesos de producción, los sistemas de consumo y en la cultura misma del diseño de indumentaria, y en vínculo con los conceptos de sostenibilidad social y ambiental, se propone que desde el diseño se puedan idear intervenciones dentro de estos sistemas, para luego recontextualizar tanto el presente como el futuro con una esperanza de reconciliación ambiente-sociedad-economía. 


\section{Análisis del Ciclo de Obsolescencia}

(...) Se ha podido comprobar que el consumidor, cuando adquiere un bien, no lo hace solamente por sus cualidades de eficiencia o funcionalidad, sino además por su significación, por lo que simboliza y por la serie de valoraciones emotivas que la posesión de ese bien expresa (Veneziani, 2012: 74).

A partir de este desarrollo sobre el ciclo de vida de una prenda y el funcionamiento de la industria del denim en Argentina, se comienzan a visibilizar algunos de los aspectos insostenibles que contiene en relación con la generación de mayor demanda y fomentar el hiperconsumo.

Para volver la mirada sobre las problemáticas puntuales que contiene la industria del denim en Argentina, en primer lugar, se debe considerar el modo de operar de su sistema para luego detectar los posibles puntos de intervención dentro de él. Tal como se mencionó anteriormente, estas prácticas de producción y consumo se basan en la implementación de ciclos de obsolescencia programada o planificada (Ver Figura 1), para poder fomentar el consumo constante y de manera masiva, gracias a la generación de aumento en la demanda.

\section{PROCESO DE LA OBSOLESCENCIA PROGRAMADA}

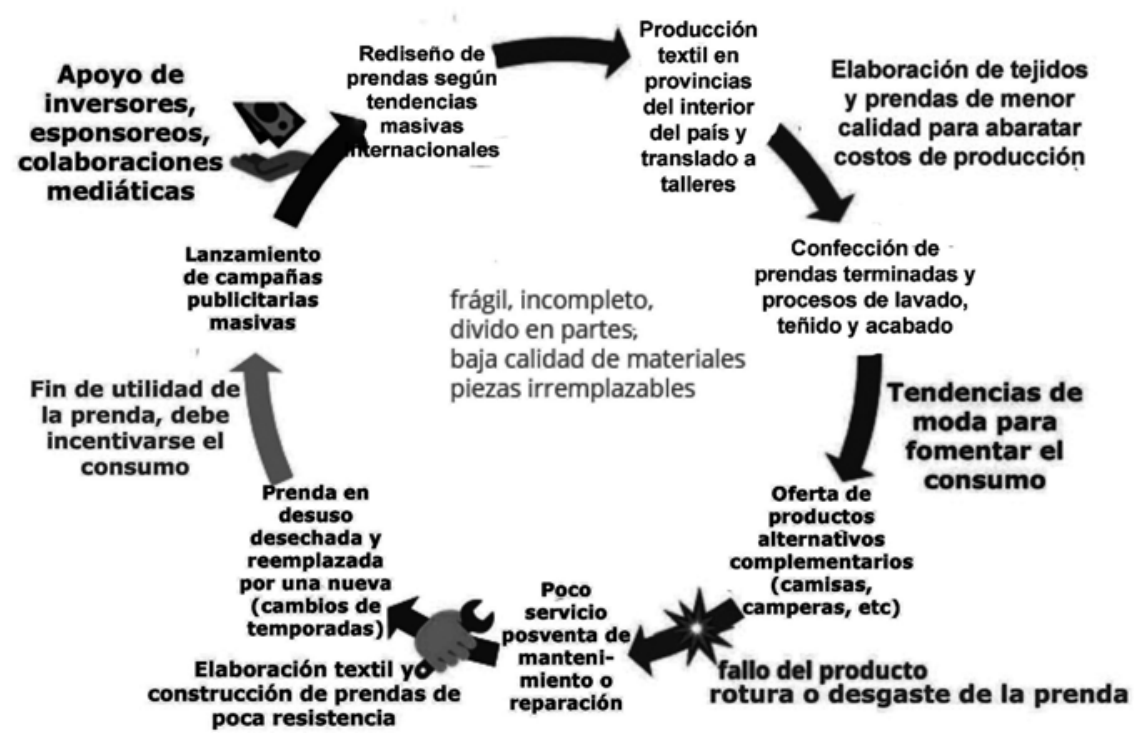

Figura 1. Representación gráfica del ciclo de obsolescencia planificada. Fuente: elaboración propia (2019). 
En estos sistemas de hiperconsumo, se fabrican los productos con la intención de acortar y limitar su vida útil para ser reemplazados por productos nuevos, permitiendo que la industria continúe el modelo de crecimiento económico. Sigue una breve descripción:

- Se lanzan campañas publicitarias con un enorme alcance gracias a los medios de comunicación masiva, que reciben el apoyo financiero de distintos inversores, esponsoreos con otras marcas y colaboraciones mediáticas. Estas campañas publicitarias se despliegan de modo masivo, a la par de que se lleva a cabo el rediseño de las prendas siguiendo las tendencias internacionales clasificadas según temporada y que se renuevan constantemente.

- Se lleva a cabo la elaboración de los textiles en plantas de producción en el interior del país para luego ser trasladados a los talleres de confección que, en su mayoría, se encuentran dentro de la provincia de Buenos Aires. Con la finalidad de abaratar costos de producción y así aumentar el margen de ganancias, no sólo se utilizan tejidos de menor calidad, sino que también se suele contratar mano de obra precarizada y se llevan a cabo las tareas en talleres (muchas veces clandestinos) con infraestructura muchas veces inadecuada, en algunos casos maquinaria desactualizada y condiciones de empleo insalubres y poco dignas.

- Una vez realizada la confección de las prendas, se aplican los debidos procesos de lavado, teñido y acabado que a menudo implican la utilización de químicos nocivos para la salud de los trabajadores y que luego terminan por contaminar los suministros de agua al no poder ser procesados correctamente, filtrándose a través de los sistemas de desagüe.

- Como método para promover mayor consumo, también se ofrecen productos complementarios alternativos dentro del rubro, como por ejemplo camisas y camperas de jean, entre otros.

- Inevitablemente, con esta limitada vida útil de los productos, debido a la utilización de tejidos de baja calidad y con menor resistencia, se producen fallos en las prendas, ya sea por roturas o por desgaste debido al uso frecuente.

- Al ofrecerse pocas alternativas de servicio posventa, de mantenimiento o de reparación de los productos, la prenda llega al final de su vida útil, haciendo que los consumidores se sientan obligados o sin otra alternativa que desecharlos para luego reemplazarlos por otros nuevos.

- Para esto debe incentivarse nuevamente el consumo, reiniciando de esta manera este ciclo de obsolescencia planificada, que funciona como un círculo vicioso.

\section{Mapeo del Wicked Problem}

(...) Todos los medios de la cultura de masas sirven para fortalecer las coacciones sociales que pesan sobre la individualidad, al excluir toda posibilidad de que el individuo se mantenga de algún modo en pie frente a la maquinaria atomizadora de la sociedad moderna (Horkheimer, citado en Croci y Vitale, 2012: 104). 
Para comprender más a fondo las problemáticas de esta industria y sus posibles causas, se plantea la elaboración del mapeo de wicked problem o problema perverso, término acuñado por Rittel y Webber en 1973. Estos problemas son considerados como una discrepancia entre el orden natural de un objeto, espacio o situación, es decir entre cómo está actualmente y como debería de estar: "La habilidad para resolver problemas perversos requiere de nuevas formas de pensar sobre el diseño, el mundo y nuestra presencia dentro de él” (Irwin, 2012:2). Para acercarse a las posibles soluciones de estos conflictos que se presentan como un entramado, resulta imprescindible incorporar la ética a través de una profunda preocupación por la sociedad y por el medioambiente. Aquí se plantea una relación de tríada en el diseño, las personas y el medioambiente, considerando lo que las personas hacen a nivel social y ecológico.

Los wicked problems tienen la particular característica de estar conformados por múltiples niveles de complejidad, involucrando a distintos actores partícipes o stakeholders, quienes usualmente tienen intereses, opiniones y sistemas de valores contrastantes. Debido a esto es que resulta casi imposible llegar a una única solución final ya que este sistema se encuentra en desequilibrio constante y en continua evolución y mutación.

Dentro del problema perverso que representa la industria del denim en Argentina, los conflictos se pueden clasificar en 5 grupos según su área de impacto y bajo las siguientes categorías: (1) Infraestructura y tecnología, (2) política y gobierno, (3) economía y finanzas, (4) ambiente y ecosistema y, finalmente, (5) relaciones sociales. Dentro de cada una de estas áreas, se desenvuelven las distintas problemáticas, que sin ser controladas, terminan causando aún más conflictos, operando bajo un efecto acumulativo, aumentando constantemente su rango de impacto razón que implica al problema perverso.

Se describen someramente los grupos por área de impacto (Ver Figura 2)

1. En lo referente a la infraestructura y tecnología, se detectan varios problemas, principalmente la falta de maquinaria especifica al rubro y la contratación de talleres textiles precarizados y con escasa o nula regulación. Sin el debido tratamiento esto puede conducir entre otras cosas, a posibles incendios, colapsos en los talleres, inundaciones por manejo inadecuado de los desechos en el sistema de desagüe, etc.

2. Bajo el paraguas de la política y gobierno, se desenvuelven muchas veces el incumplimiento de leyes laborales y ambientales, como de acuerdos de comercio que posiblemente pueden conducir a conflictos sociales, cierre de fronteras y a la inmigración ilegal, aspectos que repercuten en los sistemas de contratación, deficiencias en la legislación laboral y ambiental, ausencia de regulaciones claras, etc.

3. El aumento de la producción textil y de las ventas y el consecuente crecimiento de la industria del transporte se ubican en la categoría de economía y finanzas, y si bien parecen incorporar puntos positivos, si se dan de manera desmedida pueden finalmente causar desabastecimiento, posible cierre de mercados y el colapso de industrias sobre todo las de mediano y pequeño porte.

4. En cuanto al ambiente y el ecosistema, esta industria ha venido conduciendo a la pérdida de la biodiversidad, al contaminar los suministros de agua y polucionar el aire. Sin el debido tratamiento esto conduce a la extinción de especies, abandono de tierras infértiles $\mathrm{y}$ al agotamiento de los recursos naturales. 
5. Finalmente, bajo la categoría de relaciones sociales se desenvuelven el trabajo esclavo, la discriminación y la insalubridad, que últimamente se vinculan a la marginalización extrema, a la desigualdad de género, a una variedad de enfermedades consecuentes y a condiciones de vida infrahumanas.

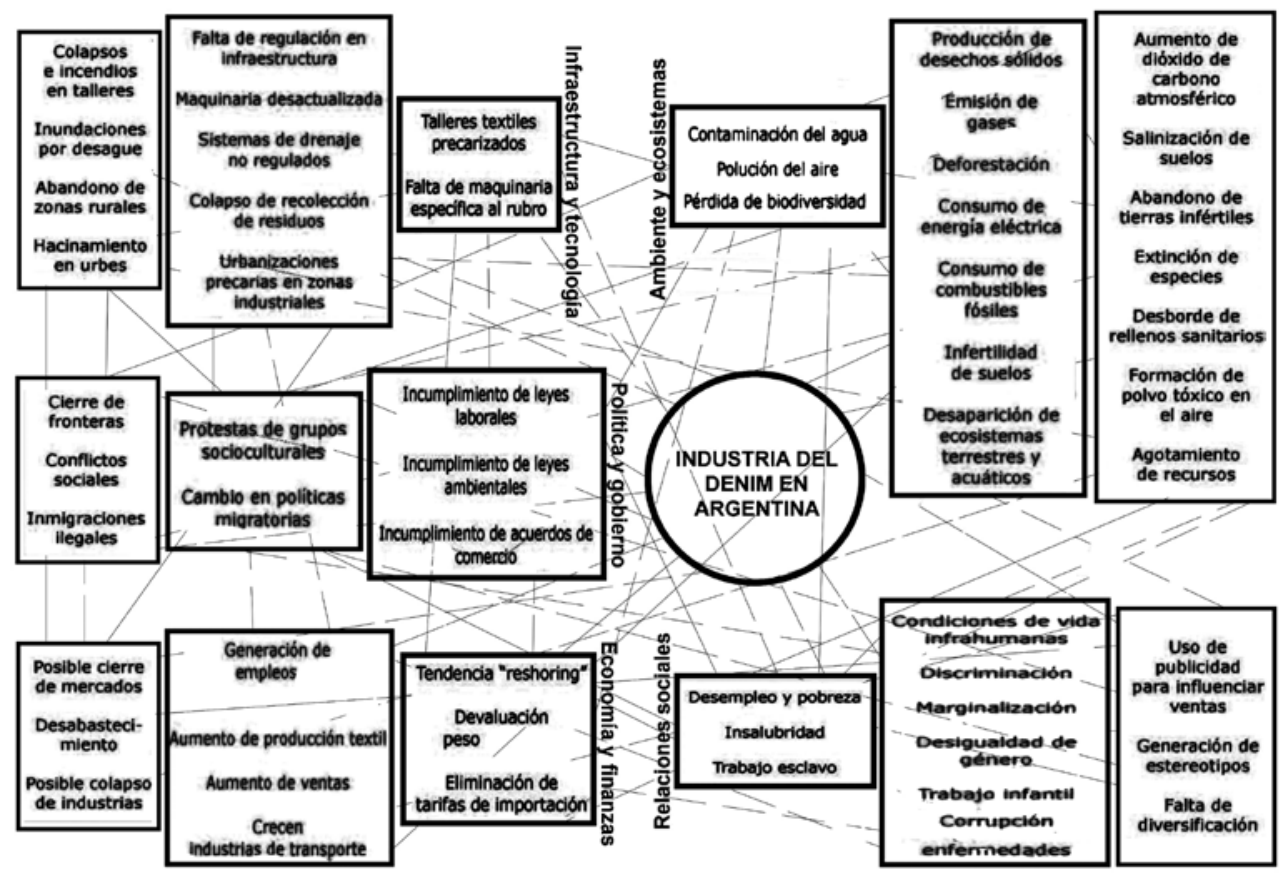

Figura 2. Mapeo síntesis del Wicked Problem (Rittel y Webber, 1973) de la industria del denim en Argentina. Fuente: elaboración propia (2019).

\section{Stakeholders y puntos de apalancamiento del sistema}

Si se consideran todas las categorías de impacto anteriormente mencionadas, dentro de cada una de ellas se puede detectar al menos un stakeholder, estos se vinculan entre sí de tres maneras: en primer lugar, a través de (a) relaciones dependientes o simbióticas, en segundo lugar, de (b) relaciones conflictivas y, en tercer lugar, de (c) relaciones mixtas. 
Haciendo foco sobre los aspectos particulares del caso de la industria del denim en Argentina se pueden nombrar como algunos de los actores intervinientes (o stakeholders) a las (1) marcas dominantes que integran esta industria, (2) los principales consumidores como por ejemplo mujeres de entre 18 a 40 años, (3) al Sindicato de Empleados Textiles, (4) al Sindicato de Empleados de Comercio, (5) a la Cámara Industrial Argentina de la Indumentaria, y (6) a la Asociación AnyBody Argentina que interviene en la lucha por la igualdad de género y por el logro de una mayor representatividad de los biotipos dentro de la moda nacional, entre otros posibles actores.

Se puede observar que todos estos grupos y entidades se sitúan dentro del sistema de manera interconectada: Por ejemplo, se podría deducir que la relación entre los consumidores y las marcas dominantes es categorizada como mixta, ya que por un lado es una relación dependiente por la generación de oferta y demanda y por el otro es una relación conflictiva debido a la falta de diversidad en los talles. En tanto los consumidores se relacionan de manera simbiótica con la Asociación AnyBody por su trabajo en la defensa por la eliminación de los estereotipos limitantes y la falta de representación de los diversos biotipos. Es relevante la labor de este tipo de asociaciones debido a que buscan visibilizar los efectos negativos y excluyentes que suelen surgir del ejercicio de esta industria. Tal como desarrolla Entwistle,

(...) cuando el cuerpo difiere de la norma de alguna manera, ya sea en términos proporcionales, de movimiento o de simetría, se convierte en un traidor de las reglas sociales y del orden natural. A este respecto, nuestra apariencia física externa se ha convertido en un importante medio de conseguir posición social (Entwistle, 2000:143).

De esta manera se observa la naturaleza compleja de las relaciones entre los stakeholders, por esto resulta necesario considerar y analizar todos los puntos de vista y posibles relaciones para poder intervenir en el sistema con la mayor eficacia posible. No sería efectivo que los diseñadores intenten imponer un orden fijo ni crear una única solución inmediata, sino que deben poder detectar los pequeños indicios de cambio, amplificarlos y posicionarse como catalizadores de esa transformación para el logro de un nuevo orden, concepto que se vincula directamente con el Diseño para la transición (Irwin, Kossoff y Tonkinwise, 2015).

En el Club de Roma (1972), Donella Meadows argumentó que toda acción llevada a cabo por un individuo o por una entidad, repercute en los demás por formar parte de un sistema en forma de red entretejida, pero en constante cambio, vinculado al por qué de la creación de un wicked problem. Dentro de los llamados problemas perversos, cada solución posible tiene sus propias ramificaciones y no existe la posibilidad de corregirla y volverla a emplear en caso de que haya fallado. Cada intento de solución implica una serie de efectos secundarios que se despliegan en forma encadenada siendo, a su vez, el síntoma o la causa del surgimiento de otros problemas perversos. Una de las formas de abordar estas problemáticas, consiste en los 12 puntos de apalancamiento o Leverage Points, sobre los cuales es posible intervenir de manera estratégica para trabajar dentro de los sistemas complejos. Debido a que realizar cambios a gran escala y de estilos de vida es considera- 
do uno de los empeños más difíciles de lograr, pero uno de los principales objetivos del Diseño para la transición, se debe apuntar a la elaboración de una propuesta a modo de intervención que pueda impulsar esos cambios en cada parte interviniente al proponer un beneficio de forma inmediata, considerado a menor escala y como el primer escalón hacia los cambios de paradigma.

Para Meadows, la capacidad de detectar y enfrentar un problema depende de cada individuo y de su ethos, es decir de su forma de ver y entender el mundo según sus ideales, creencias y valores. Debido a que todas las personas operan de diferentes modos, para poder encaminarse hacia los cambios sociotécnicos a los que apunta el Diseño para la transición, es necesario que todos los miembros de la sociedad compartan una ideología en común, fundamentada en la sostenibilidad ética y ambiental. Es necesario, además, descartar el modo de pensar lineal y reduccionista, dejando de pensar en los objetos y pasando a poner a los sujetos en primer lugar. Aquí se considera que el diseñador tiene la capacidad de visualización necesaria para alterar el paradigma, cambiando los sistemas de producción de las industrias, los estilos de vida de los consumidores y hasta el planeamiento de las ciudades, y para esto es necesario que esos cambios sean consensuados debido al vínculo indisoluble entre el diseño y la sociedad. Esta relación tan estrecha se produce ya que "la cultura del diseño como agente es una herramienta de la sociedad y a su vez, se ubica dentro de ella. Expresa una actitud, un valor y el deseo de crear mejoras" (Julier, 2000).

\section{Intervenciones sobre el sistema}

Los 12 puntos de apalancamiento propuestos por Meadows se organizan desde los más sencillos de atacar vinculado a las cifras concretas, a los más complejos de afrontar, pero a su vez los que dan mejores resultados, como el cambio de habitus, o cambios en los estilos de vida.

Para poder entender mejor sobre cuáles de estos puntos se desea trabajar, primero se debe definir cuál es la propuesta que intervendrá en el sistema y servirá como eventual catalizador del cambio. A partir del análisis previo del estado de la industria del denim en Argentina y algunas de las problemáticas que contiene, se propuso de modo contemplativo y más bien generalizado

- Un rediseño del ciclo de vida de los productos, ampliando el rango de talles tratando de eliminar la separación entre talles regulares y talles especiales.

- Esto, a su vez, potenciando una nueva experiencia de comercialización con tendencia a la inclusividad, que pueda incorporar los principios de economía circular a través de un programa de reciclado y reutilización de los descartes textiles, donde las marcas dominantes trabajarían de forma colaborativa con distintas organizaciones sin fines de lucro con la finalidad de reducir el impacto ambiental de sus procesos productivos.

De modo más detallado, lo que se propone es trabajar con una tabla de talles mucho más amplia, considerando todos los diversos biotipos que representan a sus consumidores 
para reducir las desigualdades y los sentimientos de marginalización. Además, se busca rediseñar los puntos de venta, eliminando la segregación según talles e incorporando infraestructura que contemple las normativas ambientales a través de la utilización de energías renovables y materiales reciclados y esto se acompañará con el lanzamiento de campañas publicitarias inclusivas y diversificadas, que busquen eliminar la imposición de estereotipos limitantes sobre el público. Finalmente, se les propone a los consumidores un sistema de descuentos al entregar una prenda de denim en desuso en algunos de los puntos de venta de las marcas involucradas con la finalidad de ser enviadas de forma colaborativa a una Organización No Gubernamental que trabaja con descartes textiles para crear ecobolsas o bolsas reutilizables, brindando, además, oportunidades de empleo a individuos en situación de calle o en situación de riesgo. En su totalidad, esta propuesta busca disminuir el impacto negativo de esta industria tanto en lo ético y social, como en lo ambiental, en pos de la transición hacia las visiones de futuros sostenibles.

Se considera que, de los 12 puntos de apalancamiento propuestos, este rediseño intervendría en tres:

- primero en la fuerza de retroalimentación negativa (considerado el punto número 8 en la lista de Meadows);

- en segundo lugar, en el flujo de información (siendo el punto número 6), y

- en tercer lugar, en las reglas del sistema que se ubica en el punto número 5 en la lista de Meadows. Interviene en la fuerza de retroalimentación negativa ya que de alguna manera obliga a los circuitos de poder que se ubican dentro de la industria a redefinir sus sistemas de valores al verse enfrentados a constantes y crecientes reclamos por parte de la sociedad en función de mayor diversidad e inclusividad.

Se deriva que estos reclamos surgen y vienen promovidos principalmente gracias a

(...) una generación más joven que quiere resistirse a la política más institucionalizada. Emergen como una reelaboración, una interpelación que plantea la cuestión del lugar que ocupan la estabilidad y la variabilidad. Es importante que la relación entre la comunidad marginada y la comunidad dominante no sea, estrictamente hablando, una relación de oposición (Butler, 2002: 318 y 321).

Si las marcas dominantes no se ajustan a estas tendencias, las marcas dominantes correrían el riesgo de volverse obsoletas. En este punto se interviene con la ampliación del rango de talles y el rediseño de los puntos de venta. En lo referente al flujo de información, se interviene en el lanzamiento de campañas publicitarias inclusivas, cambiando el modo en que se exhiben los cuerpos en la sociedad y creando nuevas representaciones de belleza. Esto conllevaría una modificación en la comunicación tanto verbal como visual, para los consumidores como para los productores. Finalmente, en cuanto a las reglas mismas del sistema, este rediseño intervendría incorporando los principios de economía circular, redefiniendo los hábitos de consumo de los individuos y los procesos productivos de la industria, al reducir y reutilizar los desechos textiles y al incorporar energías renovables y materiales reciclados. 
Esta propuesta de intervención impactaría por último sobre los estilos de vida al modificar las costumbres de consumo y al impactar en la salud y la sociedad. Esto guarda vinculo con la lista de necesidades esenciales propuesta por Manfred Max-Neef (1991) en su trabajo sobre las escalas del desarrollo humano o Human Scale Development, siendo estas necesidades las de subsistencia (que se satisface con abrigo, empleo), de comprensión (satisfecha con educación, comunicación), de participación (satisfecha con relaciones sociales), de creación (que se satisface con el arte y el diseño) y finalmente de identidad (satisfecha a través de la representación del género, las costumbres, etc.). Max-Neef clasifica a estas necesidades como fundamentales para la vida humana, finitas y globales, es decir que son iguales en todas las culturas del mundo.

Como resultado de esto, se considera que en el contexto inmediato resultan afectados los usuarios, los diseñadores y los comerciantes y productores. En el contexto intermedio son afectados los espacios públicos, los negocios y la industria local, mientras que en el contexto amplio son afectados los mercados, las instituciones, las ciudades, provincias y países y finalmente las normas, los valores y la cultura misma.

Situando esta propuesta de rediseño en el mapeo de Pathways of Social Design, propuestos por el Instituto Winterhouse en 2013 (Ver figura 3), se considera que, al incorporar el rango de experiencia de los diseñadores, de los equipos interdisciplinarios y de los grupos sociales en las distintas escalas de compromiso (desde la intervención, a la innovación y últimamente llegando a la transformación) se pueden catalizar los cambios sociotécnicos necesarios para transicionar hacia las visiones de futuro sostenible.

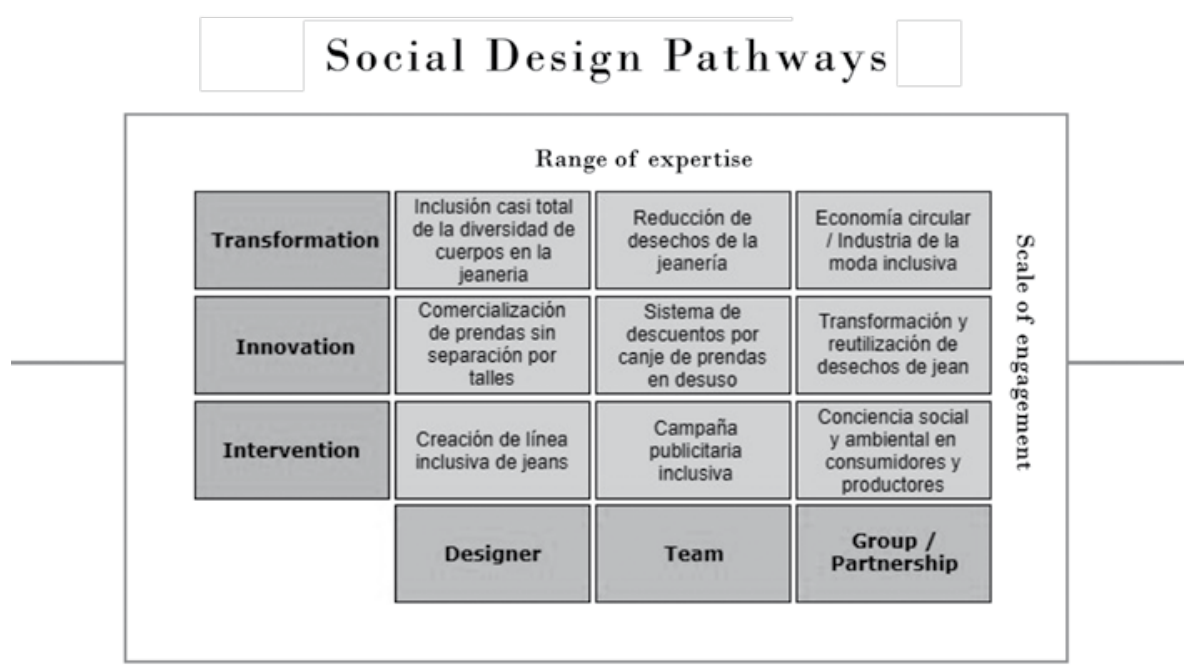

Figura 3 (arriba) y 4 (p. 159). Representación gráfica de la propuesta de rediseño según los Pathways of Social Design (Winterhouse, 2013), y camino síntesis de la propuesta. Fuente: elaboración propia (2019). 


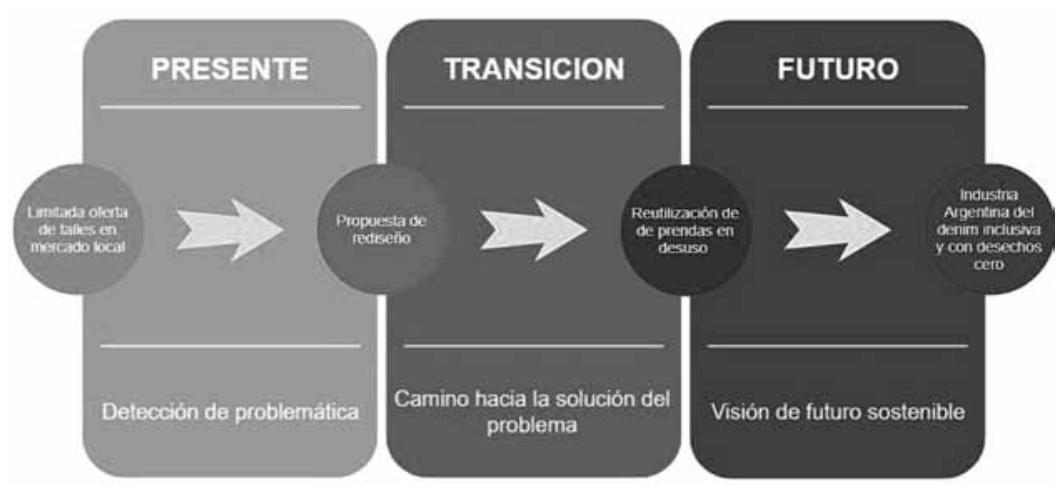

Figura 4 .

Por último, si se consideran algunas de las reglas de diseño ecológico de Orr (2018), tales como la eliminación de desperdicios, el hacerlo accesible, el diseñar para el desarmado y la reparación, la máxima participación pública y también el hacerlo bello, se considera que esta propuesta de intervención tiene la potencialidad de cumplir con todos estos puntos. Mencionando una vez más el proceso cíclico del Diseño para la transición, de diseñar y luego aplicar intervenciones; esperar, observar y luego recontextualizar el presente y el futuro a partir de los resultados obtenidos, se establece que el presente de la industria del denim en Argentina refleja la problemática de una limitante oferta de talles, una experiencia de compra segregada, un corto ciclo de vida de las prendas y un manejo inadecuado de los residuos.

Para ello se planteó esta propuesta de ideas para un rediseño analizando la industria del denim como parte de una posible intervención en el sistema, que permitan que sea inclusiva, tendiente a desechos cero y fundamentada en la economía circular.

\section{Referencias bibliográficas}

Bauman, Z. (2000). Modernidad líquida. Buenos Aires: Fondo de Cultura Económica de Argentina, S.A.

Barreiro, A. (2004). La construcción social del cuerpo en las sociedades contemporáneas. La Coruña: Universidad de La Coruña.

Butler, J. (2002). Cuerpos que importan: sobre los límites materiales y discursivos del sexo. Buenos Aires: Editorial Paidós SAICF. 
Butler, J. (2007). El género en disputa. El feminismo y la subversión de la identidad. Barcelona: Ediciones Paidós Ibérica S.A.

Cline, E. (2014). Moda desechable: El escandaloso costo de la ropa barata. México D.F.: Ediciones Culturales Paidós.

Croci, P. y Vitale, A. (2012). Los cuerpos dóciles: hacia un tratado sobre la moda. (3era edición). Buenos Aires: La Marca Editorial.

Entwistle, J. (2002). El cuerpo y la moda: una visión sociológica. Barcelona: Editorial Paidós. Irwin, T. (2012). Wicked Problems and the Relationship Triad. Edinburgo: Floris Books.

Julier, G. (2000). The culture of design. Londres: Sage Publications Ltd.

Julier, G. (2006). From visual culture to design culture. Cambridge: Massachusetts Institute of Technology.

Max-Neef M, Elizalde A, y Hopenhayn M. (1991). Human Scale Development. Lanham: The Apex Press.

Oittana, L. (2013). La desaparición de lo real o el éxtasis de la comunicación. Rosario: Universidad Nacional de Rosario.

Pampliega de Quiroga A. y Pichon-Riviere E. (1985). Psicología de la vida cotidiana. Buenos Aires: Editorial Nueva Visión SAIC.

Rittel H. y Webber M. (1973). Dilemmas in a General Theory of Planning. Amsterdam: Elsevier Scientific Publishing Company.

Sainz, C. (2017). El poder oculto del jean. Disponible en https://www.clarin.com/sociedad/ poder-oculto-jean_0_r1hRCDWTx.html, Recuperado el 10/07/2019.

Veneziani, M. (2012). Moda, economía y sociedad. Buenos Aires: Editorial Nobuko.

Vizcarra, F. (2002). Premisas y conceptos básicos en la sociología de Pierre Bourdieu. Colima: Universidad de Colima.

Vizcarra, F. y Olvalle, L. (2011). Ciberculturas: el estado actual de la investigación y análisis. Santiago: Pontificia Universidad Católica de Chile.

Zambrini, L. (2008). Cuerpos, indumentarias y expresiones de género: el caso de las travestis en la Ciudad de Buenos Aires. En Figari, C., Jones, D. y Pecheny, M. (Comp.), (2008). Todo sexo es político. Estudios sobre sexualidades en Argentina. Buenos Aires: Libros del Zorzal.

S/D. Donella Meadows archives. Leverage Points: Places to intervene in a System. Disponible en http://donellameadows.org/archives/leverage-points-places-to-intervene-in-a-system/. Recuperado el 25/08/2019.

S/D. Winterhouse Institue Social Design Pathways. Disponible en http://www.winterhouse institute.org/pathways. Recuperado el 25/08/2019.

\begin{abstract}
At present, the need to sustain a paradigm shift in the production and marketing systems of the denim industry has become evident, working on concepts such as circular economy and Design for Transition (Irwin, Kosoff and Tonkinwise, 2015) in order to turn fashion into a sustainable practice. Based on a critical analysis of the scenario and using the conceptual framework of Transition Design, we seek to contribute ideas for an intervention proposal using the leverage points proposed by Donella Meadows (1997).
\end{abstract}


Keywords: Transition Design - Leverage Points - Wicked Problems - Circular Economy Denim Industry - Clothing Design - Sustainable Design - Sustainability.

Resumo: Atualmente, a necessidade de sustentar uma mudança de paradigma nos sistemas de produção e comercialização da indústria de jeans tornou-se evidente, trabalhando em conceitos como economia circular e Design for Transition (Irwin, Kosoff e Tonkinwise, 2015) para transformar a moda em uma prática sustentável. Com base em uma análise crítica do cenário e usando a estrutura conceitual do Design for Transition, buscamos contribuir com idéias para uma proposta de intervenção usando os pontos de alavancagem propostos por Donella Meadows (1997).

Palavras chave: Design de transição - pontos de alavancagem - problemas perversos economia circular - indústria de jeans - design de roupas - design sustentável - sustentabilidade.

[Las traducciones de los abstracts fueron supervisadas por el autor de cada artículo] 